\title{
Inferior Vena Cava Filter as a Preventive Device for Recurrent Pulmonary Embolism in a Patient with Nephrotic Syndrome
}

\author{
Yu-Che Tsai ${ }^{a} \quad$ Yuan-Kai Hsieh $^{\mathrm{b}}$ Chien-Te Lee $^{\mathrm{a}}$ \\ Divisions of ${ }^{a}$ Nephrology and ${ }^{b}$ Cardiology, Department of Medicine, Chang Gung Memorial Hospital-Kaohsiung \\ Medical Center, Chang Gung University College of Medicine, Kaohsiung, Taiwan, ROC
}

\section{Key Words}

Greenfield filter · Nephrotic syndrome $\cdot$ Pulmonary embolism

\begin{abstract}
Objective: This study describes a patient with nephrotic syndrome who experienced recurrent pulmonary emboli as renal disease relapsed frequently. Clinical Presentation: A 30 year-old male, who was diagnosed as having nephrotic syndrome at 12 , presented with general edema, ascites and hypoalbuminemia. Although the patient responded well to steroid therapy, a common cold frequently caused flare-up of nephrotic syndrome. Histopathological examination of renal tissue revealed minimal disease change. At 17, the patient had a first pulmonary embolism attack with concurrent upper respiratory tract infection. Full-blown manifestations of nephrotic syndrome with low antithrombin III levels were identified at that time. Anticoagulant therapy was administered to treat the pulmonary embolism. Since the first attack, several episodes of pulmonary emboli occurred, which, combined with kidney disease, triggered refractory nephrotic syndrome. The nephrotic syndrome did not remit, and a secondary renal biopsy identified glomerular pathological changes similar to previous biopsy results. To prevent recurrent pulmonary emboli, a Greenfield inferior vena cava filter was implanted in April 2002. No further pulmonary embolism events occurred after interventional filter deployment. Conclusion: The results of this study suggest that in-
\end{abstract}

ferior vena cava filter implantation can be effective for preventing recurrent pulmonary embolism complicating refractory nephrotic syndrome. Copyright $\odot 2007$ S. Karger AG, Basel

\section{Introduction}

Minimal change disease accounts for $90 \%$ of nephrotic syndrome cases in children [1]. Thromboembolic events are critical complications for nephrotic syndrome patients. Hypercoagulability is the principle mechanism that activates thromboembolism. Among the underlying abnormalities are coagulating pathway factors, cofactors, fibrinogen, the fibrolytic system, clotting inhibitors and platelet function [2]. Pulmonary embolism (PE) is the most severe and life-threatening complication, accounting for $8 \%$ of all thromboembolic events of complication [2]. Conventional anticoagulation therapy reduces the recurrence of PE. However, recurrent PE is usually refractory to oral anticoagulants and has a high mortality rate [3]. The present study describes a 30-year-old male patient diagnosed as having steroid-dependent minimal change disease with recurrent PE. After a Greenfield inferior vena cava (IVC) filter had been implanted, no further PE attack occurred, despite the recurrence of nephrotic syndrome. Life-threatening PE was effectively prevented via an implanted IVC filter.

\section{KARGER}

Fax +4161306 1234

E-Mail karger@karger.ch

www.karger.com
(C) 2007 S. Karger AG, Basel

$1011-7571 / 07 / 0164-0321 \$ 23.50 / 0$

Accessible online at:

www.karger.com/mpp
Dr. Chien-Te Lee

Division of Nephrology, Department of Internal Medicine

Chang-Gung Memorial Hospital, Kaohsiung, 123 Ta-Pei Road

Niao-Sung Hsiang, Kaohsiung Hsien 833, Taiwan (ROC)

Tel. +886 731 7123, ext. 8306, Fax +8867 732 2402, E-Mail ctlee33@adm.cgmh.org.tw 


\section{Case Report}

A previously healthy 12 -year-old male was admitted to the pediatric ward at Chang-Gung Memorial Hospital in 1987 due to progressive swelling of both eyelids and decreased urine volume for almost 1 week. He denied any recent upper respiratory tract infections and his medical history was unremarkable.

The results of physical examination were normal except for swollen eyelids and lower leg edema. No lymphoadenopathy or neurological abnormality was observed. Urinalysis identified heavy proteinuria and no red blood cell casts. The hemogram results indicated a white blood cell count of $14.35 \times 10^{9} / 1$ and hemoglobulin of $15.9 \mathrm{gm} / \mathrm{dl}$. Biochemical data were as follows: serum albumin $=2.4 \mathrm{~g} / \mathrm{dl}$; total protein $=4 \mathrm{~g} / \mathrm{dl}$; blood nitrogen urea $=6.0 \mathrm{mmol} / \mathrm{l}$; creatinine $=88.4 \mu \mathrm{mol} / \mathrm{l}$; cholesterol $=15.5$ $\mathrm{mmol} / \mathrm{l}$; glutamate oxaloacetate transaminase $=32 \mathrm{U} / \mathrm{l}$; glutamate pyruvate transaminase $=16 \mathrm{U} / \mathrm{l}$; and alkaline phosphatase $=$ $20 \mathrm{U} / \mathrm{l}$. No evidence of recent streptococcus infection was identified. The antistreptolysin $\mathrm{O}$ level was within normal range. Negative antinuclear antibody titer and normal C3 and C4 levels excluded the possibility of an immunological disorder. Virus markers did not indicate hepatitis B or C. High-dose steroids were administered on the supposition of primary minimal change disease. Limb edema and proteinuria resolved soon and remained in remission for 1 year. However, nephrotic syndrome recurred 2 years later with similar presentations. The patient again received high-dose steroids and responded well and rapidly to the treatment. The nephrosis, however, recurred frequently and a kidney biopsy confirmed a diagnosis of minimal change disease.

In December 1992, the patient had sudden onset of dyspnea with pleuritic chest pain. A series of examinations identified increased arterial-alveolar gradient $(73.5 \mathrm{~mm} \mathrm{Hg})$, low antithrombin level $(0.011$ SI, normal range $=0.8-1.2)$, with normal ECG tracing and cardiac enzymes. Technetium-99m macroaggregate on lung perfusion scan demonstrated unilateral decreased perfusion in the left lung field, indicating PE. No renal vein thrombosis, or intracardiac or intrapulmonary artery thrombosis was detected by kidney or cardiac echo. Venous Doppler study found no evidence of venous occlusion. During this episode, heparinization was administered initially; then the regimen was shifted to warfarin and life-long anticoagulation therapy was prescribed thereafter.

From 17 to 26 years of age, the patient had $6 \mathrm{PE}$ attacks despite maintenance and targeted international normalization ratio. Each episode was resolved by administering unfractionated heparin with concomitant increases in the steroid regimen. No other site had simultaneous thromboembolism. To identify other likely causes for his recurrent PE, his family history was reviewed; no inherited thromboembolic disorders existed. Additionally, his protein $\mathrm{C}$ and S levels were normal and the result of an antiphospholipid antibody was negative. No hyperhomocysteinemia was found. Subsequent antithrombin III levels were in the lower limit of the normal range and thus, antithrombin concentrate was not prescribed. In the face of recurring renal disease with its severe complication of PE, a second kidney biopsy was performed to examine the probable transformation and determine whether the aggressiveness of the treatment should be increased. The pathological report engendered the same diagnosis of minimal change disease. To prevent further PE during nephrotic syndrome flare-up, a Greenfield IVC filter was implanted in 2002. No renal vein thrombosis was identified by angiography during surgery, and therefore, the IVC filter was implanted below the renal vein. No PE attacks occurred during nephrotic syndrome relapse following filter implantation. A series of lung perfusion scans detected a defect over the right upper lung field, possibly indicating some old insults. However, the patient's pulmonary function test was normal.

The patient currently takes low doses of prednisolone, cyclosporine and warfarin for PE prophylaxis and a lipid-lowering agent for hypercholesterolemia. Although the underlying glomerulopathy still fluctuates, this patient has had no recurrent $\mathrm{PE}$ after Greenfield IVC filter implantation.

\section{Discussion}

Minimal change disease, the most common etiology of nephrotic syndrome in children, generally responds well to steroid therapy. Over $50 \%$ of children administered steroids remit within 2 weeks, and more than $90 \%$ of children achieve complete remission within 8 weeks. The initial attack should be treated with prednisone or prednisolone 60 $\mathrm{mg} / \mathrm{m}^{2} /$ day for $4-6$ weeks. For treatment of patients with steroid-dependent minimal change disease, $2 \mathrm{mg} / \mathrm{kg} /$ day of cyclophosphamide for 12 weeks or $6 \mathrm{mg} / \mathrm{kg} /$ day of cyclosporine (for children) and $5 \mathrm{mg} / \mathrm{kg} /$ day (for adults) for various periods has been suggested [4]. The patient here presented initially with steroid-sensitive minimal change disease, which was determined to be steroid-dependent, as the disease did not resolve completely. In view of the recurrent nephrotic events, this patient underwent secondary renalbiopsy, indicating steroid-dependent minimal change disease without any superimposed or transformed lesions. In these circumstances, cyclosporine therapy was initiated, but it did not produce long-term remission.

In patients with nephrotic syndrome, the incidence of venous thrombosis typically outnumbers arterial thrombosis [5]. Increased incidence of thrombosis is strongly associated with hypercoagulability. The hypercoagulable state in nephrotic syndrome is characterized by low zymogen factors (factors IX, XI, XII), a substantial increase in cofactors (factors V and VIII), an increase in plasma fibrinogen levels, a reduction in the levels of antithrombin III and antiplasmin activity and thrombocytosis, increased platelet aggregation and an increase in $\beta$-thromboglobulin levels [2]. In addition to these disease-associated acquired defects, concurrent volume depletion, hemoconcentration and administration of corticosteroids can precipitate thromboembolism in such patients. Although the possible contribution of steroids in this patient cannot be ruled out, PE attacked as a flare-up of renal disease, and at that time the prednisolone dosage was generally maintained at a low dose. Nevertheless, therapy for underlying glomerular disease is the mainstay for pre- 
venting complications associated with nephrotic syndrome. It has been noted that long courses of anticoagulation therapy benefit patients with recurrent $\mathrm{PE}$ and deficiencies in antithrombin III, protein C and protein S [6].

Specifically, this patient had a low antithrombin III level. In patients with nephrotic syndrome, acquired deficiency and urinary excretion of antithrombin III has been demonstrated [7]. This is particularly likely since the molecular weight of human antithrombin III $(62,000$ Da) closely compares with that of albumin, the urinary loss and reduced serum levels of which characterize nephrotic syndrome. This patient had acquired deficiency of antithrombin III in active glomerulopathy that was normalized as underlying renal disease in remission. At follow-up, the antithrombin III level remained at the lower level of the normal range. This finding highlights the probable contribution of antithrombin III in recurrent PE observed in this patient.

With an active hemorrhage or recurrent PE despite intensive and prolonged anticoagulation therapy, implantation of an IVC filter has been suggested [6]. The fact that this patient had already been on long-term prophylactic warfarin treatment since the first episode and still experienced recurrent PE suggested a need for IVC filter implantation. Trousmon first proposed the notion of blocking PE coming from venous thrombi [8]. The IVC filter then became available and effectively prevented PE. Greenfield and Proctor [9] noted that indications of IVC filter implantation were contradictory to anticoagulant therapy (45\%), recurrent PE (20\%) and complications of anticoagulant therapy (16\%) [9]. In their series, $50 \%$ of patients had concurrent deep vein thrombosis, and only 1 patient had underlying renal disease. Follow-up study demonstrated that the Greenfield filter decreased the recurrent $\mathrm{PE}$ rate to $4 \%$ with an IVC patency rate of $96 \%$ [9]. Although deep vein thrombosis was not evident when $\mathrm{PE}$ was diagnosed in this patient, implantation of an IVC filter was indicated as small thrombi may have been neglected and thrombi within the lung vessel accumulated gradually. Long-term outcome and complications of permanent IVC filter implantation are important. Underlying disease and concurrent use of anticoagulants can determine the efficacy and safety of permanent IVC filters [8].

A Medline search identified only 1 report [10] describing a patient with renal disease complicated by recurrent PE successfully treated by Greenfield filter implantation. In that study, a 40 -year-old male with membranous glomerulonephritis developed nephrotic syndrome. Semioccluded thrombus in the right renal vein with concomitant $\mathrm{PE}$ was diagnosed via renal venography. That patient suffered from recurrent PE under strong anticoagulation therapy upon admission. The implantation of a Greenfield filter effectively prevented PE, with no further episodes occurring. Conversely, the patient in the present study had recurrent $\mathrm{PE}$ over the years with fluctuating renal disease activity. This study and that reported earlier [10] involved implantation of a Greenfield filter; however, the Greenfield filter was implanted at different locations based on the presence and absence of renal vein thrombosis. Moreover, the underlying glomerular disease and clinical course were substantially different for the 2 cases.

\section{Conclusion}

This study, for the first time, describes a patient with steroid-dependent minimal change disease who developed nephrotic syndrome and clinically recurrent PE that was prevented by implantation of a Greenfield filter. We suggest that IVC filter implantation may be an effective means of preventing recurrent $\mathrm{PE}$ that complicates refractory nephrotic syndrome.

\section{References}

$>1$ Fogo AB: Minimal change disease and focal segmental glomerulosclerosis. Nephrol Dial Transplant 2001;16:74-76.

2 Llach F: Hypercoagulability, renal vein thrombosis, and other thrombotic complications of nephrotic syndrome. Kidney Int 1985;28:429-439.

$\checkmark 3$ Carson JL, Kelley MA, Duff A: The clinical course of pulmonary embolism. N Engl J Med 1992;326:1240-1245.

4 Bargman JM: Management of minimallesion glomerulonephritis: evidence-based recommendations. Kidney Int 1999;70:S3-S16.
5 Chuang CH, Lee CT, Cheng YF, Huang TL, Hung KH, Chen JB: Bilateral renal infarctions and lower limbs artery thrombosis in a patient with nephrotic syndrome. J Nephrol 2004;17:311-315.

$\checkmark 6$ Goldhaber SZ: Pulmonary embolism. N Engl J Med 1998;339:93-104.

7 Vaziri ND: Acquired deficiency and urinary excretion of antithrombin III in nephrotic syndrome. Arch Intern Med 1984;144:18021803.

8 Decousus H, Leizorovicz A, Parent F, Page Y, Tardy B, Girard P, Laporte S, Faivre R, Char- bonnier B, Barral FG, Huet Y, Simonneau G: A clinical trial of vena caval filters in the prevention of pulmonary embolism in patients with proximal deep-vein thrombosis. New Engl J Med 1998;338:409-415.

$\checkmark 9$ Greenfield LJ, Proctor MC: Twenty-year clinical experience with Greenfield filter. Cardiovasc Surg 1995;3:199-205.

10 O’Brien AA, O’Donnell JP, Keogh JA: Renal vein thrombosis with recurrent pulmonary emboli in the nephrotic syndrome: use of Greenfield filter. Postgrad Med J 1986;62: 223-225. 